Portland State University

PDXScholar

\title{
Data From: Microplastic Concentrations in Two Oregon Bivalve Species: Spatial, Temporal, and Species Variability
}

\author{
Britta Baechler \\ Portland State University, baechler@pdx.edu \\ Elise F. Granek \\ Portland State University, graneke@pdx.edu \\ Matthew V. Hunter \\ Oregon Department of Fish and Wildlife \\ Kathleen E. Conn \\ United States Geological Survey
}

Follow this and additional works at: https://pdxscholar.library.pdx.edu/esm_data

Part of the Environmental Health and Protection Commons, Environmental Indicators and Impact Assessment Commons, and the Environmental Monitoring Commons Let us know how access to this document benefits you.

\section{Recommended Citation}

Baechler, Britta; Granek, Elise F.; Hunter, Matthew V.; and Conn, Kathleen E., "Microplastic Concentrations in Two Oregon Bivalve Species: Spatial, Temporal, and Species Variability" (2019). [Dataset].

https://doi.org/10.15760/esm-data.1

This Dataset is brought to you for free and open access. It has been accepted for inclusion in Environmental Science and Management Datasets by an authorized administrator of PDXScholar. Please contact us if we can make this document more accessible: pdxscholar@pdx.edu. 
Table 1. Description of the fields needed to describe the creation of your dataset.

\begin{tabular}{|c|c|}
\hline Title of dataset & $\begin{array}{l}\text { Microplastic Concentrations in Two Oregon Bivalve Species: Spatial, } \\
\text { Temporal, and Species Variability }\end{array}$ \\
\hline URL of dataset & $\begin{array}{l}\text { Data is available in the Portland State University PDXScholar data } \\
\text { repository at: https://doi.org/10.15760/esm-data.1 }\end{array}$ \\
\hline Abstract & $\begin{array}{l}\text { Microplastics are an ecological stressor with implications for } \\
\text { ecosystem and human health when present in seafood. We } \\
\text { quantified microplastic types, concentrations, anatomical burdens, } \\
\text { geographic distribution, and temporal differences in Pacific oysters } \\
\text { (Crassostrea gigas) and Pacific razor clams (Siliqua patula) from } 15 \\
\text { Oregon coast, U.S.A. sites. Organisms were chemically digested and } \\
\text { visually analyzed for microplastics, and material type was determined } \\
\text { using Fourier Transform Infrared Spectroscopy. Microplastics were } \\
\text { present in organisms from all sites. On average, whole oysters and } \\
\text { razor clams contained } 10.95 \pm 0.77 \text { and } 8.84 \pm 0.45 \text { microplastic } \\
\text { pieces per individual, or } 0.35 \pm 0.04 \text { and } 0.16 \pm 0.02 \text { pieces g-1 tissue, } \\
\text { respectively. Contamination was quantified but not subtracted. Over } \\
\text { 99\% of microplastics were fibers. Spring samples contained more } \\
\text { microplastics than summer in oysters but not razor clams. This study } \\
\text { provides a spatially extensive baseline of microplastics in Oregon } \\
\text { bivalves and is the first to determine Pacific razor clam } \\
\text { concentrations. }\end{array}$ \\
\hline Keywords & $\begin{array}{l}\text { Microfiber, microplastic, Pacific razor clam, Pacific oyster, Crassostrea } \\
\text { gigas, Siliqua patula }\end{array}$ \\
\hline Lead author for the dataset & Britta Baechler, Dr. Elise Granek \\
\hline $\begin{array}{l}\text { Title and position of lead } \\
\text { author }\end{array}$ & $\begin{array}{l}\text { Britta Baechler, PhD student; Dr. Elise Granek; Professor, Portland } \\
\text { State University }\end{array}$ \\
\hline $\begin{array}{l}\text { Organization and address } \\
\text { of lead author }\end{array}$ & $\begin{array}{l}\text { Portland State University } \\
\text { Environmental Science\& Management } \\
\text { PO Box } 751 \\
\text { Portland, OR } 97207\end{array}$ \\
\hline $\begin{array}{l}\text { Email address of lead } \\
\text { author }\end{array}$ & baechler@pdx.edu \\
\hline $\begin{array}{l}\text { Additional authors or } \\
\text { contributors to the dataset }\end{array}$ & $\begin{array}{l}\text { Britta R. Baechler }{ }^{1} \text {, Elise F. Granek }{ }^{1} \text {, Matthew V. Hunter }{ }^{2} \text {, Kathleen E. } \\
\text { Conn }{ }^{3}\end{array}$ \\
\hline
\end{tabular}

\footnotetext{
${ }^{1}$ This document liberally borrows from a similar document provided by the Environmental Data Initiative 


\begin{tabular}{|c|c|}
\hline & $\begin{array}{l}{ }^{1} \text { Portland State University, Portland, OR; }{ }^{2} \text { Oregon Department of Fish } \\
\text { and Wildlife, Astoria, OR; }{ }^{3} \text { United States Geological Survey, Tacoma, } \\
\text { WA }\end{array}$ \\
\hline $\begin{array}{l}\text { Organization associated } \\
\text { with the data }\end{array}$ & $n / a$ \\
\hline Funding & $\begin{array}{l}\text { PI: Dr. Elise Granek; Project title: Microplastics in our food? Providing } \\
\text { a baseline for microplastic concentrations in the tissues of Oregon's } \\
\text { commercially and recreationally important Pacific oysters and razor } \\
\text { clams; Funding: Oregon Sea Grant \# NA14OAR4170064 }\end{array}$ \\
\hline License & $\begin{array}{l}\text { This work is licensed under a Creative Commons Attribution- } \\
\text { NonCommercial-NoDerivatives } 4.0 \text { International License }\end{array}$ \\
\hline $\begin{array}{l}\text { Geographic location - } \\
\text { verbal description }\end{array}$ & $\begin{array}{l}15 \text { sites along the Oregon coast, USA (9 razor clam sites, } 6 \text { oyster } \\
\text { grower sites) }\end{array}$ \\
\hline $\begin{array}{l}\text { Geographic coverage } \\
\text { bounding coordinates }\end{array}$ & $46^{\circ} 11^{\prime} 42.15^{\prime \prime} N, 123^{\circ} 59^{\prime} 38.31 \mathrm{~W}, 42^{\circ} 27^{\prime} 15.78^{\prime \prime} N, 124^{\circ} 25^{\prime} 35.12^{\prime \prime} W$ \\
\hline Time frame - Begin date & $4 / 2017$ \\
\hline Time frame - End date & $3 / 2019$ \\
\hline General study design & $\begin{array}{l}\text { Pacific oysters and razor clams were collected from } 15 \text { sites during } \\
\text { low tide series in spring (April 27-28) and summer (July 21-31), } 2017 . \\
\text { Whole oysters were purchased from growers at six grower sites } \\
\text { during both seasons. One oyster grower was selected from each of } \\
\text { six Pacific oyster-producing bays. In this report, oyster grower names } \\
\text { are withheld and are coded randomly as OY1-OY6. Razor clams were } \\
\text { collected from nine sandy beach sites stretching from Clatsop in the } \\
\text { north, to Gold beach. Of the nine clam sites, reported by name, four } \\
\text { were sampled in both spring and summer, providing a temporal } \\
\text { snapshot of microplastic frequencies. All samples were transported } \\
\text { to the Applied Coastal Ecology laboratory at Portland State University } \\
\text { (PSU) in Portland, Oregon. }\end{array}$ \\
\hline Methods description & $\begin{array}{l}\text { All samples were transported on ice to the Applied Coastal Ecology } \\
\text { laboratory at Portland State University (PSU) in Portland, Oregon, in } \\
\text { clean } 2 \mathrm{~L} \text { glass Mason jars. Shell and tissue measurements were } \\
\text { collected with a digital Mitutoyo caliper and Ohaus balance accurate } \\
\text { to } 0.01 \mathrm{~mm} \text { and } 0.01 \mathrm{~g} \text {, respectively. Bivalve shells were rinsed with } \\
\mathrm{DI} \text { water to remove sand, mud, and debris, were shucked into clean } \\
120 \mathrm{~mL} \text { Mason jars and frozen at }-20 \text {-C. } \\
\text { Samples were thawed and digested for } 24 \mathrm{~h} \text { in a laminar flow fume } \\
\text { hood using } 10 \% \text { potassium hydroxide }(\mathrm{KOH}) \text {. Digestion began with } \\
\text { the first organism from each site and season, then proceeded to the } \\
\text { second organism from each site, until all samples were processed. } \\
\text { Samples were poured through a } 7.6 \mathrm{~cm} \text { diameter, } 63 \text { mm stainless }\end{array}$ \\
\hline
\end{tabular}




\begin{tabular}{|c|c|}
\hline & $\begin{array}{l}\text { steel sieve. Material retained on the sieve was rinsed into clean, } \\
\text { labeled glass petri dishes. Petri dishes with Petristickers }{ }^{\circledR} \text { affixed to } \\
\text { the bases were placed in a drying oven at } 40 \text { o } \mathrm{C} \text { for } 24 \mathrm{~h} \text { and stored in } \\
\text { sealed tubs prior to microscope processing. Due to high levels of } \\
\text { organic material and sand granules remaining in clam samples after } \\
\text { initial digestion, a second } 10 \% \mathrm{KOH} \text { digestion combined with } \\
\text { hypersaline density separation ( } 330 \mathrm{~g} / \mathrm{L} \text { Fisher Chemical Certified ACS } \\
\text { Crystalline NaCl) was utilized. Samples were analyzed under a Leica } \\
\text { M165C stereomicroscope ( } 10-120 x \text { magnification) connected via a } \\
\text { Leica IC } 80 \text { HD camera to a computer running Leica Application Suite X } \\
\text { imaging software. }\end{array}$ \\
\hline $\begin{array}{l}\text { Laboratory, field, or other } \\
\text { analytical methods }\end{array}$ & $\begin{array}{l}\text { NAME: Each individual specimen was assigned a label for } \\
\text { identification after being brought from the field to the PSU lab } \\
\text { (Naming convention: SITE-SEASON-SPECIES-SAMPLE NUMBER- } \\
\text { SAMPLE TYPE) } \\
\text { SPECIES: Species (Pacific razor clam or Pacific oyster) was determined } \\
\text { based on existing knowledge } \\
\text { SAMTYPE: Type of sample was recorded and coded for each } \\
\text { individual specimen [C=control, CW= whole razor clam, CT= razor } \\
\text { clam tissue only, CG= razor clam gut only, OW= whole Pacific oyster, } \\
\text { OT=oyster tissue only, OG= oyster gut only, PB=procedural bkank, } \\
\text { SA= saline density separation step (razor clams only)] } \\
\text { Sample: Type of sample was recorded (in full text) for each individual } \\
\text { Specimen [Control=Control; Whole= whole sample (gut \& tissue), } \\
\text { GUT= gut only sample, TISSUE= tissue only sample, Proced. Blank= } \\
\text { procedural blank, Saline= saline density separation step (razor clams } \\
\text { only)] } \\
\text { SITE: Sample location was recorded at the date and time of } \\
\text { collection. Site names were written for razor clam sites but were } \\
\text { coded as OY1-OY6 for Pacific oysters for grower privacy. (OY1-OY6: } \\
\text { Oyster site (randomized). CLA= Clatsop Beach; CAN= Cannon Beach; } \\
\text { MEA= Cape Meares; AGA= Agate Beach; NEW= Newport South } \\
\text { Beach; COO= Bayside North Spit, Coos Bay; BDF= Bastendorff Beach; } \\
\text { WHS= Whiskey Creek; GDB= Gold Beach.) } \\
\text { SEASON: Season sample was harvested or purchased in during 2017 } \\
\text { was recorded at time of harvest or purchase. (SP= Spring; SUM= } \\
\text { Summer) } \\
\text { and accurate to 0.01mm }\end{array}$ \\
\hline
\end{tabular}




\begin{tabular}{|c|c|}
\hline & 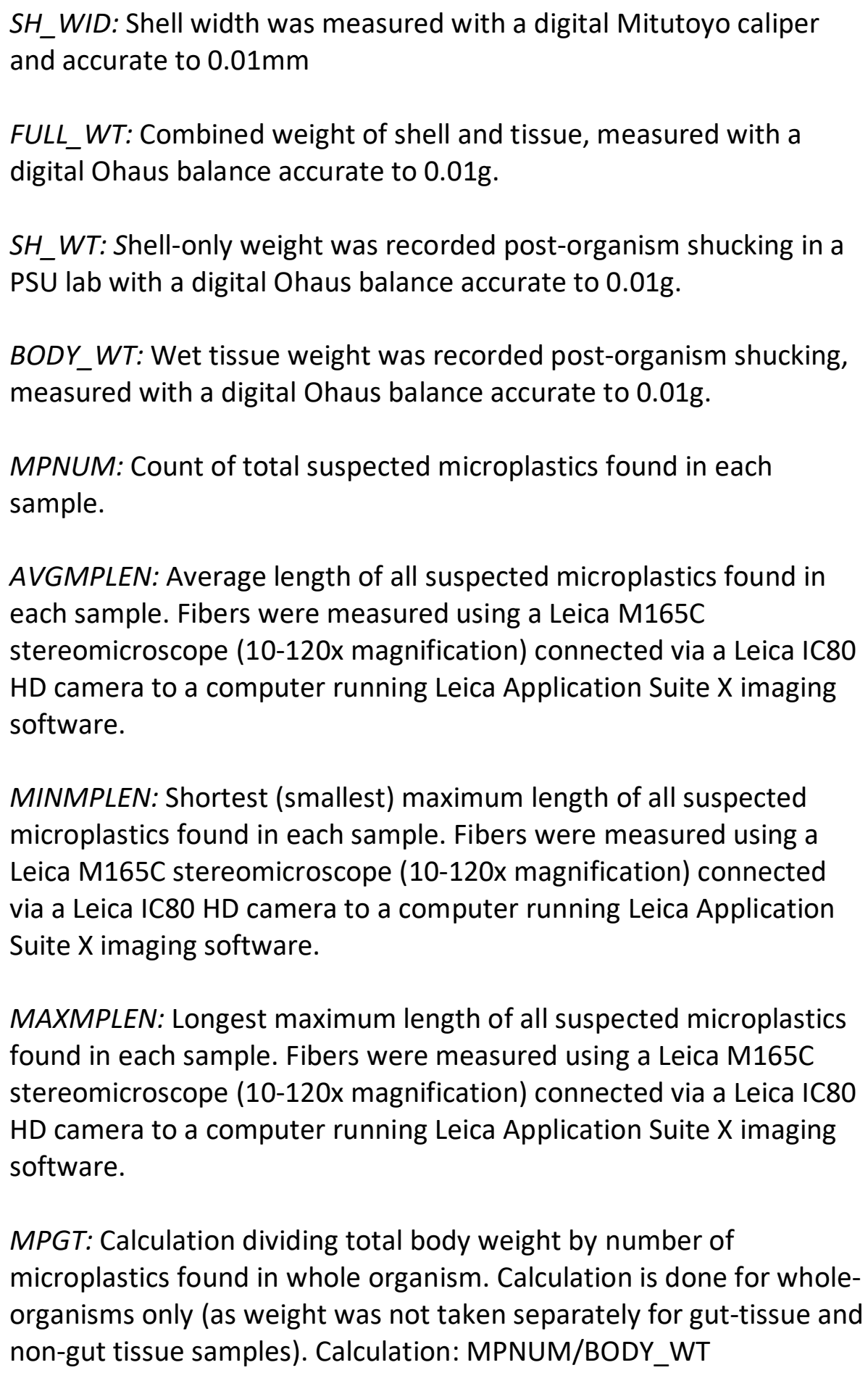 \\
\hline $\begin{array}{l}\text { Taxonomic species or } \\
\text { groups }\end{array}$ & Pacific razor clam (Siliqua patula), Pacific oyster (Crassostrea gigas) \\
\hline Quality control & $\begin{array}{l}\text { One hundred percent cotton clothing, cotton lab coats, and nitrile } \\
\text { gloves were worn at all times during sample processing, digestion, } \\
\text { and analysis procedures. All shucking implements and glassware } \\
\text { were rinsed three times with deionized (DI) water filtered to } 0.22 \\
\mu \mathrm{m} \text {. To quantify procedural contamination, } 11 \text { replicates of } 50 \mathrm{~mL}\end{array}$ \\
\hline
\end{tabular}




\begin{tabular}{|c|c|}
\hline & $\begin{array}{l}\text { filtered DI water were frozen in } 4 \text { oz jars and underwent the same } \\
\text { digestion and analysis process as organism samples. One procedural } \\
\text { blank per week was chemically digested alongside field samples on a } \\
\text { randomly generated day. Additionally, three procedural blanks were } \\
\text { collected to quantify contamination introduced by the secondary } \\
\text { digestion and hypersaline density separation of razor clam samples. } \\
\text { During microscope analysis, a petri dish containing filtered DI water } \\
\text { was placed adjacent to each sample on the microscope base and left } \\
\text { open to the air to quantify airborne contaminants. After sample } \\
\text { analysis, the control petri dish was analyzed for microplastics; any } \\
\text { particles detected were assumed to be contamination and were } \\
\text { measured and categorized. } \\
\text { Aside from microplastic contamination prevention quality control, } \\
\text { data were reviewed for accuracy. }\end{array}$ \\
\hline Additional information & $n / a$ \\
\hline
\end{tabular}

Table 2. Data dictionary: description of the variables (i.e., columns) in EACH dataset. You must provide sufficient detail for another user to understand and use the data. If there are 10 variables (i.e., columns) in the dataset, then there should be 10 rows in this table that describe each column. Be sure to include all relevant information for your dataset, including the unique identifiers for your dataset or system, dates, replicate numbers, latitude and longitude of sampling locations, etc.

Dataset filename: MP_Data_Baechler et al_2019.xls

Dataset description: For each sample organism: Collection location and season, sample type, species, Biological measurements, number of microplastics, average microplastic length, smallest and largest microplastic detected. 


\begin{tabular}{|c|c|c|c|c|c|}
\hline Column name & Description & Units & Code explanation & Data format & Mtssing data code \\
\hline NAME & $\begin{array}{l}\text { Organism sample } \\
\text { number }\end{array}$ & $n / a$ & $\begin{array}{l}\text { Naming convention: STE- } \\
\text { SEASON-SPECES- } \\
\text { SAMPYE NUMBER- } \\
\text { SAMPYE TYPE }\end{array}$ & $\begin{array}{l}\text { [Site: OY1-OY6: Oyster site (randomized). CLA= Clatsop } \\
\text { Beach; CAN= Cannon Beach; MEA= Cape Meares; AGA= Agate } \\
\text { Beach; NEW = Newport South Beach; COO= Bayside North } \\
\text { Spit, Coos Bay, BDF= Bastendorff Beach; WHS= Whiskey } \\
\text { Creek; GDB= Gold Beach.][Season: SP= Spring; SUM= } \\
\text { Surnmer][Species: R= razor clam; OY= oyster][Sample } \\
\text { number: Organism number for site][Sample type: } \\
\text { Whole=blank; Gut=-G; Tissue=--T, Procedural blank= PB] }\end{array}$ & blank cell \\
\hline SPECIES & Species of sample & $n / a$ & $\begin{array}{l}\mathrm{C}=\text { control; Clam=Pacific } \\
\text { razor clam; Oyster= } \\
\text { Pacific oyster; PB= } \\
\text { procedural blank, SA= } \\
\text { saline density separation } \\
\text { step (razor clams only) }\end{array}$ & [see code explanation] & blank cell \\
\hline SAMTYPE & $\begin{array}{l}\text { Type of sample: } \\
\text { Species-specific }\end{array}$ & $n / a$ & $\begin{array}{l}\mathrm{C}=\text { control, } \mathrm{CW}=\text { whole } \\
\text { razor clam, } \mathrm{CT}=\text { razor } \\
\text { clam tissue only, } \mathrm{CG}= \\
\text { razor clam gut only, } \\
\text { OW= whole Pacific } \\
\text { oyster, OT= oyster tissue } \\
\text { only, OG= oyster gut } \\
\text { only, } \mathrm{PB}=\text { procedural } \\
\text { blank, SA= sal ine } \\
\text { density separation step } \\
\text { (razor clams only) }\end{array}$ & [see code explanation] & blank cell \\
\hline Sample & $\begin{array}{l}\text { Type of sample: } \\
\text { Not species- } \\
\text { specific }\end{array}$ & $n / a$ & $\begin{array}{l}\text { Control=Control; } \\
\text { Whole= whole sample } \\
\text { (gut \& tissue), GUT= gut } \\
\text { only sample, TSSUE= } \\
\text { tissue only sample, } \\
\text { Proced. Blank= } \\
\text { procedural blank, } \\
\text { Saline= saline density } \\
\text { separation step (razor } \\
\text { clams only) }\end{array}$ & [see code explanation] & blank cell \\
\hline SחE & $\begin{array}{l}\text { Site sample was } \\
\text { collected from }\end{array}$ & $n / a$ & $\begin{array}{l}\text { OY1-OY6: Oyster site } \\
\text { (randornized). CIA= } \\
\text { Clatsop Beach; CAN= } \\
\text { Cannon Beach; MEA= } \\
\text { Cape Meares; AGA= } \\
\text { Agate Beach; NEW= } \\
\text { New port South Beach; } \\
\text { COO= Bayside North } \\
\text { Spit, Coos Bay; BDF= } \\
\text { Bastendorff Beach; } \\
\text { WHS= Whiskey Creek; } \\
\text { GDB= Gold Beach. }\end{array}$ & [see code explanation] & blank cell \\
\hline SEASON & $\begin{array}{l}\text { Season sample } \\
\text { was collected in }\end{array}$ & $n / a$ & $\begin{array}{l}\text { SP= Spring; SUM= } \\
\text { Summer }\end{array}$ & [see code explanation] & blank cell \\
\hline SH_LEN & Shell length & $\mathrm{mm}$ & $n / a$ & number & blank cell \\
\hline SH_WID & Shell width & $\mathrm{mm}$ & n/a & number & blank cell \\
\hline FUL_WT & $\begin{array}{l}\text { Full weight of shell } \\
\text { and body (wet } \\
\text { weight) }\end{array}$ & E & $n / a$ & number & blank cell \\
\hline SH_WT & $\begin{array}{l}\text { Weight of shells } \\
\text { only }\end{array}$ & $\mathbf{E}$ & $n / a$ & number & blank cell \\
\hline BODY_WT & $\begin{array}{l}\text { Weight of body } \\
\text { tissue only (wet } \\
\text { weight) }\end{array}$ & E & $n / a$ & number & blank cell \\
\hline MPNUM & $\begin{array}{l}\text { Number of } \\
\text { microplastics in } \\
\text { sample }\end{array}$ & $n / a$ & $n / a$ & count & błank cell \\
\hline AVGMPLEN & $\begin{array}{l}\text { Average } \\
\text { microplastic length } \\
\text { for sample }\end{array}$ & $\mathrm{mm}$ & $n / a$ & average number & blank cell \\
\hline MIN MPYEN & $\begin{array}{l}\text { Smallest } \\
\text { microplastic found } \\
\text { in sample }\end{array}$ & $\mathrm{mm}$ & $n / a$ & number & blank cell \\
\hline MAXMPIEN & $\begin{array}{l}\text { Largest } \\
\text { microplastic found } \\
\text { in sample }\end{array}$ & $\mathrm{mm}$ & $n / a$ & number & blank cell \\
\hline MPGT & $\begin{array}{l}\text { Number of } \\
\text { microplastics per } \\
\text { gram of orgnaism } \\
\text { tissue }\end{array}$ & $n / a$ & $\begin{array}{l}\text { Calculation of } \\
\text { MPNUM/BODY_WT. } \\
\text { Will be left blank if no } \\
\text { body weight was } \\
\text { collected. }\end{array}$ & number & blank cell \\
\hline
\end{tabular}


Table 3. Data provenance

If you used data derived from other sources, provide the information here so future users know where the data came from. N/A

Notes and Comments: N/A 